\title{
Actitudes y Disposición de Estudiantes de la Salud y Ciencias Sociales en la Atención Clínica a Personas Trans
}

\author{
Attitudes and Dispositions of Health and Social Science Students in the Clinical \\ Care of Trans Persons
}

\begin{abstract}
Isabella Peña Buitrago ${ }^{1}$, Luisa María Cubides Madroñero ${ }^{2}$, Leydi Lorena Hoyos Chalarca ${ }^{3}$, \& Paula Andrea Hoyos Hernández $4^{*}$

1 Pontificia Universidad Javeriana, Cali, Colombia. (D) https://orcid.org/0000-0002-1048-9765

2 Pontificia Universidad Javeriana, Cali, Colombia. (D) https://orcid.org/0000-0003-3722-9602

3 Pontificia Universidad Javeriana, Cali, Colombia. (ID https://orcid.org/0000-0001-5206-3706

4 Pontificia Universidad Javeriana, Cali, Colombia. (D) http://orcid.org/0000-0001-7948-6338

* Correspondencia: paulahoyos@javerianacali.edu.co
\end{abstract}

Recibido: 29 septiembre 2021 | Aceptado: 10 diciembre 2021 | Publicado: 19 diciembre 2021

$$
\text { WWW.REVISTACARIBENADEPSICOLOGIA.COM }
$$

\section{Citar como:}

Peña Buitrago, I., Cubides Madroñero, L. M., Hoyos Chalarca, L. L., \& Hoyos Hernández, P. A. (2021). Actitudes y disposición de estudiantes de la salud y ciencias sociales en la atención clínica a personas trans. Revista Caribeña de Psicología, 5, e5963. https://doi.org/10.37226/rcp.v5i1.5963

\section{RESUMEN}

El objetivo de esta investigación fue identificar la relación entre las actitudes, la disposición y la edad en la atención clínica a personas trans por parte de estudiantes universitarios. Esto se hizo con una muestra de 185 estudiantes de facultades de salud y ciencias sociales en Colombia a través de un cuestionario de aplicación virtual constituido por ítems de dos escalas diseñadas por Esteban et al. (2020) y Rodríguez-Madera et al. (2017). Se obtuvo que la gran mayoría de los y las participantes no presentan prejuicios altos o disposición hacia la población trans. Además, las disposiciones se relacionan con la edad debido a que las personas mayores a 30 años presentaron menores niveles de prejuicios y disposición. Finalmente, se concluye que las actitudes variarán por factores como la presión social y la intención de cambiar ese comportamiento. En cuanto a las disposiciones, se deben tener en cuenta el contexto social del/la profesional y las herramientas que usa para adquirir conocimientos en temas de diversidad sexual y de género.

Palabras Claves: actitudes, atención clínica, disposición, personas trans

\section{ABSTRACT}

The objective of this research was to identify the relationship between attitudes, disposition and age in the clinical care of trans people by university students. This was done with a sample of 185 students from health and social sciences faculties in Colombia through a virtual application questionnaire consisting of items from two scales designed by Esteban et al. (2020) and Rodríguez-Madera et al. (2017). It was obtained that the vast majority of participants do not present high prejudices or disposition towards the trans population. In addition, dispositions are related to age because people older than 30 years presented lower levels of prejudice and disposition. Finally, it is 
Actitudes y Disposición de Estudiantes de la Salud y Ciencias Sociales en la Atención Clínica a Personas Trans

concluded that attitudes will vary by factors such as social pressure and the intention to change that behavior. Regarding dispositions, the social context of the professional and the tools he/she/them uses to acquire knowledge on issues of sexual and gender diversity should be taken into account.

Keywords: attitudes, clinical care, disposition, trans persons

\section{INTRODUCCIÓN}

La atención en salud en el contexto colombiano sigue teniendo grandes desafíos (Vargas et al., 2010). Los pocos estudios existentes en poblaciones con identidades de género trans coinciden en afirmar que las dificultades se acentúan mucho más dada las diversas barreras estructurales y contextuales a las que se exponen las personas con identidades de género no hegemónicas durante el proceso de la atención en salud (Fondo de Población de las Naciones Unidas [UNFPA], 2019).

Estudios en países como Estados Unidos, España, Argentina, Cuba y Chile concuerdan en que las barreras que perciben el personal de la salud y las poblaciones trans son las siguientes: el desconocimiento de las necesidades particulares de la población (Barrientos et al., 2019; Chárriez y Seda, 2015; Chisolm et al., 2018; Fajardo, 2017; Farji, 2018; Francia-Martínez et al., 2017; Gleason et al., 2016; Godoy et al., 2013; Marques et al., 2015); el acompañamiento de los profesionales de la salud sin considerar el enfoque de género, la formación profesional en atención a personas trans deficiente (Gonzáles, 2020a; Gonzáles, 2020b; Gonzáles y Novoa, 2019; La Perra Roja, 2019; Losada, 2017; Mendoza, 2019; Rueda, 2019); la patologización a partir de los manuales de clasificación de padecimientos médicos como la Clasificación Internacional de Enfermedades o CIE y el Manual Diagnóstico y Estadístico de los Trastornos Mentales o DSM; la poca promoción de servicios preventivos de los servicios médicos y la estigmatización acerca de portar y transmitir enfermedades e infecciones (como el VIH).

Considerando lo anterior, las barreras que más prevalecen en la atención médica de esta población empuja a muchas/os/es a la clandestinidad y automedicación (Aylagas-Crespillo et al., 2017; Campo et al., 2014; Chárriez y Seda, 2015; Domínguez et al., 2018; Estrada y García, 2010; Fajardo, 2017; UNFPA, 2019; Gleason et al., 2016; Mejía y Benavides, 2008; Lasso, 2014; Padilla et al., 2016; Palma y Orcasita, 2019; Profamilia y Estado del Reino de los Países Bajos
[Profamilia], 2019; Ritterbusch et al., 2018; Roberts y Fantz, 2014; Snelgrove et al., 2012).

Los y las profesionales en salud reconocen la falta de capacitación clínica en atención a la población LGBT y poca formación que se les da acerca de la diversidad sexual. No obstante, hay algunos/as/es que están abiertos/as/es a aprender sobre su correcta atención, teniendo en cuenta las necesidades y particularidades de la población; esto independiente de sus valores y principios personales enmarcados en posturas cisheteronormativas y religiosas (Williams-Colón et al., 2021). Por otro lado, otros/as/es profesionales presentan prejuicios y actos discriminatorios hacia esta población (Antoniucci, 2016; Builes et al., 2017; Chisolm et al., 2018; Farji, 2018; Francia-Martínez et al., 2017; Hoyos-Hernández y Valderrama, 2020; Palma y Orcasita, 2019; Vance et al., 2015; Vizcaíno et al., 2019; Zaldúa et al., 2015; Zapata et al., 2019).

Ahora, los estudios realizados en Colombia proponen recomendaciones tanto metodológicas para futuras investigaciones, como médicas en cuanto a la atención en salud de esta población. En cuanto a las metodológicas, los/las/les autores/as/es plantean que realizar estudios de diferentes diseños alrededor de las nuevas construcciones acerca de las orientaciones sexuales e identidades de género resultaría socialmente y científicamente pertinente. Asimismo, se evidenció que la mayoría de los artículos encontrados abordan la temática desde una mirada principalmente cualitativa, haciendo uso de las entrevistas y grupos focales, siendo minoritarios los estudios de tipo cuantitativo (Campo et al., 2014; Domínguez et al., 2018; Hoyos-Hernández y Valderrama, 2020; Lasso, 2014; Mejía y Benavides, 2008; Sacsa, 2019; Profamilia, 2019). Igualmente, los estudios colombianos reconocen que dichas barreras en salud, junto con las sociales, educativas y familiares que presentan las personas trans, afectan negativamente su salud y bienestar (Berredo, 2011; Ritterbusch et al., 2018).

Es importante destacar que el gobierno nacional de Colombia ha implementado diferentes políticas nacionales, como la de Prestación de Servicios de 
Salud que tiene como propósito "garantizar el acceso, optimizar el uso de los recursos y mejorar la calidad de los servicios que se prestan a la población" (Ministerio de Salud y Protección Social, 2005, p.17). Sin embargo, se ha evidenciado que, en general, la población colombiana presenta dificultades a la hora de acceder a los servicios de salud (Vargas et al., 2010).

En Colombia existen políticas públicas específicas que velan por los derechos de las personas trans, como la Política Nacional de Sexualidad, Derechos Sexuales y Derechos Reproductivos (Ministerio de Salud y Protección Social, 2014). Además, existe una guía de atención titulada Lineamientos de atención en los servicios de salud que consideran el enfoque diferencial de género y no discriminación para las personas LGBTI de la Fundación de Población de las Naciones Unidas (UNFPA, 2019). Esta guía resulta relevante para la presente investigación puesto que dentro de estos lineamientos se encuentran estipulados las pautas o direccionamientos que se deben tener en cuenta a la hora de brindar atención en salud a las personas trans dependiendo del servicio solicitado; todo esto transversal a la argumentación y sustentación de la creación de estos lineamientos para las personas LGBTI en general (UNFPA, 2019).

Además, en Colombia se han desarrollado diferentes estrategias para abordar la atención en salud de las personas trans, promoviendo que tengan una atención integral y de excelente calidad. Estos son los servicios transafirmativos (Colombia Diversa, 2010) y el protocolo titulado ¿Cómo adaptar los servicios de salud sexual y reproductiva a las necesidades y circunstancias de las personas con experiencia de vida trans? (Profamilia, 2019). Adicionalmente, tiene un marco estratégico llamado Modelo Integral de Atención en Salud (MIAS), que pretende velar por el bienestar de la población teniendo en cuenta todas sus necesidades y tratando de abordarlas de manera parcial. Este modelo parte de la Política de Atención Integral en Salud (PAIS) del Ministerio de Salud (2016) declarada en la Ley 1751 del 2016 y cuenta con numerosos componentes que le permiten velar por su cumplimiento, entre los cuales se encuentran las Rutas Integrales de Atención en Salud (RIAS). Estas rutas son un instrumento que definen las condiciones necesarias para garantizar la atención integral en salud a personas, familias y comunidades (Ministerio de Salud, 2016).
Por último, es importante resaltar que ninguna de las investigaciones revisadas plantea la diferencia entre los momentos del curso de vida de una persona, el cual es un factor determinante para analizar las actitudes favorables de los/las/les profesionales de la salud y ciencias sociales en atención a personas trans. Lo anterior deja un vacío investigativo que se considera relevante teniendo en cuenta la época sociocultural en la que está inmerso el mundo actualmente; en esta, las nuevas generaciones pueden estar más sensibilizadas y con mayor apertura ante la diversidad de género. Se considera lo anterior debido a que la diversidad sexual y de género en la cultura colombiana tiene mayor visibilidad, pues ahora se evidencian más grupos activistas, comunidades y movimientos sociales.

En este sentido, en la presente investigación se planteó el objetivo de identificar la relación entre las actitudes, la disposición y la edad en la atención clínica a personas trans por parte de estudiantes universitarios. Teniendo en cuenta lo anterior, se estableció la siguiente hipótesis: a menor edad y trayectoria clínica, mayor disposición y actitudes positivas en los procesos de atención en salud a personas trans. Se plantea esto debido a que las investigadoras del presente estudio consideran que los profesionales más jóvenes están más permeados y sensibilizados ante la diversidad de género que los que tienen más experiencia clínica.

En este orden de ideas, la actitud se entenderá a partir de Ubillos et al. (2004), los cuales reúnen los aportes más significativos de autores como Allport en 1935, Vallerand en 1994 y Zimbardo y Leippe en 1991, quienes consideraban la actitud como un estado de disposición mental y nerviosa, organizado mediante la experiencia y que ejerce un influjo directivo dinámico en la respuesta del individuo a toda clase de objetos y situaciones. Por su parte, Lilienfeld et al. (2011) mencionan que las actitudes se originan en creencias o ideas que las personas tienen, y que les permiten dar un valor negativo o positivo a las diferentes situaciones. Es por esto por lo que las actitudes juegan un papel fundamental en la manera en cómo las personas se relacionan con el entorno (Esteban et al., 2020).

En esta misma línea, es importante mencionar que el presente estudio tendrá en cuenta el modelo tradicional que menciona que la actitud tiene tres 
componentes que la definen: cognitivo, que se compone de ideas articuladas, pensamientos y creencias sobre un objeto, evento, persona o grupo social; afectivo, que hace referencia a las emociones y sentimientos que se tienen respecto al objeto; y conductual, que consiste en los comportamientos que se realizan con relación al objeto, los cuales pueden presentarse en conductas de acercamiento o distanciamiento (Gutiérrez, 2015; Lancheros et al., 2007). Cabe resaltar que un componente puede presentarse en mayor medida que el otro, por lo que la intensidad de las actitudes puede variar. Ante esto, se ha comprobado que, si se puede aumentar el sentimiento de agrado o bienestar frente a algo en particular, hay mayor probabilidad de que influya en el comportamiento hacia el evento, persona o grupo social (Esteban et al., 2020).

Respecto a disposición, esta es la descripción del organismo en cuanto a estar listo a realizar una conducta determinada (Glosario análisis conductual, 2014) que, en este caso, sería estar dispuesto a brindar la atención en salud a la población trans. Y, en el caso del presente estudio, la disposición a proporcionar servicios a personas trans se entiende como una disposición positiva para brindar atención de salud trans-sensible (Rodríguez-Madera et al., 2017).

Por su parte, la persona transgénero se define como aquella que vive un género diferente al sexo asignado al nacer (Xavier et al., 2005) y que puede tener también tratamientos médicos y transformaciones corporales o no (APA, 2011; Hoyos y Concha, 2020; Rodríguez-Madera et al., 2017; UNFPA, 2019). Para efectos de esta investigación, la persona transgénero será concebida como aquella persona inconforme con su género asignado al nacer y que, por lo tanto, vive en un género distinto a este, teniendo la posibilidad de realizar tratamientos médicos y transformaciones corporales si así lo desea.

\section{MÉTODO}

\section{Diseño de Investigación y Consideraciones Éticas}

El presente estudio tuvo un diseño transversal correlacional (Hernández et al., 2014) y se ajustó a las normativas de protección de datos de Colombia, a la declaración de Helsinki y la normativa ética en Colombia sobre principios éticos en la investigación con seres humanos. A partir de la resolución 8430 del
Ministerio de Salud del 2013, se considera como una investigación de riesgo menor al mínimo.

\section{Participantes}

La muestra constó de 185 personas entre estudiantes y egresados/as de programas de pregrado y posgrado de facultades de salud y de ciencias sociales de Colombia con experiencia en atención clínica y/o investigativa en comunidades trans de mínimo 4 meses.

\section{Instrumentos}

Se utilizó una encuesta compuesta de dos instrumentos distintos y un cuestionario de datos sociodemográficos construido por las investigadoras. Los instrumentos usados fueron: la Escala de Actitudes de Psicoterapeutas hacia la Comunidad Transgénero (Esteban et al., 2020) y la Escala sobre Disposición Transgénero (Rodríguez-Madera et al., 2017). Estos instrumentos midieron actitudes y disposición, respectivamente.

\section{Procedimientos Generales y Análisis de Datos}

La encuesta se construyó y se aplicó de manera virtual por motivos de emergencia sanitaria por COVID-19, a través del aplicativo Google Forms, el cual contenía las consideraciones éticas de la investigación y el consentimiento informado antes de iniciar la encuesta. Para la distribución de la encuesta se construyó una pieza gráfica y se envió la invitación a través de redes académicas y sociales. Quienes estuvieron interesados en participar en la investigación, accedieron a través del link de Google y, una vez consideraban que cumplían con los criterios de inclusión, aceptaban concediendo su consentimiento para tales fines y procedían a diligenciar la encuesta. Esta tuvo una duración de aproximadamente 15 minutos y no solicitaba ningún dato de identificación. Los resultados fueron analizados por medio del Paquete Estadístico SPSS versión 20.0 para Windows usando estadística descriptiva e inferencial.

\section{RESULTADOS}

En este apartado se presentarán los resultados obtenidos de la aplicación de la Escala de Actitudes de Psicoterapeutas hacia la Comunidad Transgénero (Esteban et al., 2020) y la Escala sobre Disposición Transgénero (Rodríguez-Madera et al., 2017). Lo anterior con el fin de dar cuenta del objetivo de identificar las relaciones entre las actitudes y la edad en la 
atención clínica a personas trans por parte de estudiantes universitarios. Primero, se mostrarán las características de las personas que participaron para continuar con la correlación entre la edad y la atención clínica y, finalmente, la correlación entre las actitudes, la edad y la atención clínica.

\section{Caracterización de las Personas Participantes}

La caracterización de los y las participantes del estudio se muestra en la Tabla 1. La muestra del estudio se conformó por 185 personas, de las que el 73,5\% es menor de 30 años. De estas, el 72,4\% fueron mujeres y el $71,9 \%$ se identifica con el género femenino. Además, las orientaciones sexuales de los y las participantes variaron entre heterosexualidad $(81,6 \%)$, homosexualidad $(9,7 \%)$, bisexualidad $(6,5 \%)$ y en el $2,1 \%$ restante se encuentran la pansexualidad y demisexualidad.

\section{Tabla 1}

Índices de Discriminación y Varianza Explicada de los Ítems de la Escala de Autoeficacia en el Trabajo

\begin{tabular}{lc}
\hline \multicolumn{1}{c}{ Variable } & $n(\%)$ \\
\hline Sexo & $134(72,4)$ \\
Mujer & $51(27,6)$ \\
Hombre & \\
Género & $133(71,9)$ \\
Femenino & $52(28,1)$ \\
Masculino & \\
Orientación Sexual & $51(81,6)$ \\
Heterosexual & $18(9,7)$ \\
Homosexual & $12(6,5)$ \\
Bisexual & $3(1,6)$ \\
Pansexual & $1(0,5)$ \\
Demisexualidad & \\
Afiliación religiosa & $106(57,3)$ \\
Católica & $17(9.2)$ \\
Cristiana & $1(0,5)$ \\
Protestante & $1(0,5)$ \\
Mormona & $9(4,9)$ \\
Ateo & $19(10,3)$ \\
Agnosticismo & $6(3,2)$ \\
Otra & $26(14,1)$ \\
Ninguna de las anteriores & \\
Enalidad el Valle del Cauca & $161(87)$ \\
Fuera del Valle del Cauca & $24(13)$ \\
&
\end{tabular}

\begin{tabular}{|c|c|}
\hline Variable & $n(\%)$ \\
\hline \multicolumn{2}{|l|}{ Edad } \\
\hline Menores de 30 años & $136(73,5)$ \\
\hline Mayores de 30 años & $49(26,5)$ \\
\hline \multicolumn{2}{|l|}{ Programas de pregrado } \\
\hline Medicina & $54(29,2)$ \\
\hline Psicología & $83(44,9)$ \\
\hline Enfermería & $9(4,9)$ \\
\hline Trabajo social & $9(4,9)$ \\
\hline Fisioterapia & $9(4,9)$ \\
\hline Sociología & $1(0.5)$ \\
\hline Terapia ocupacional & $10(5,4)$ \\
\hline Odontología & $9(4,9)$ \\
\hline Fonoaudiología & $1(0,5)$ \\
\hline \multicolumn{2}{|l|}{ Posgrado } \\
\hline Especializaciones y maestrías & $53(28,6)$ \\
\hline Sin posgrado & $132(71,4)$ \\
\hline \multicolumn{2}{|l|}{ Formación en temas de diversidad sexual } \\
\hline Se ha formado & $99(53,5)$ \\
\hline No se ha formado & $86(46,5)$ \\
\hline \multicolumn{2}{|l|}{$\begin{array}{l}\text { Métodos de formación en temas de diver- } \\
\text { sidad sexual y de género }\end{array}$} \\
\hline Instituciones gubernamentales & $26(14,1)$ \\
\hline Instituciones educativas & $49(26,5)$ \\
\hline Plataformas digitales & $39(21,1)$ \\
\hline ONG o fundaciones & $25(13,5)$ \\
\hline Otro & $15(8,1)$ \\
\hline $\begin{array}{l}\text { No tiene formación en temas de } \\
\text { diversidad sexual y de género }\end{array}$ & $90(48,6)$ \\
\hline \multicolumn{2}{|l|}{ Tiempo de experiencia clínica } \\
\hline Menos de 4 años & $125(67,6)$ \\
\hline Más de 4 años & $60(32,4)$ \\
\hline \multicolumn{2}{|l|}{$\begin{array}{l}\text { Lugar en el que ha brindado atención clí- } \\
\text { nica }\end{array}$} \\
\hline Clínica & $73(39,5)$ \\
\hline Hospital & $74(40)$ \\
\hline ONG & $41(22,2)$ \\
\hline Institución gubernamental & $19(10,3)$ \\
\hline Institución educativa universitaria & $31(16,8)$ \\
\hline Institución básica primaria & $16(8,6)$ \\
\hline Consulta particular & $44(23,8)$ \\
\hline Trabajo independiente & $15(8,1)$ \\
\hline Investigación & $30(16,2)$ \\
\hline Otra & $7(3,8)$ \\
\hline \multicolumn{2}{|l|}{ Atención a personas trans } \\
\hline Sí ha atendido & $107(57,8)$ \\
\hline No ha atendido & $78(42,2)$ \\
\hline
\end{tabular}


En cuanto a la localidad, el $87 \%$ vive en el Valle del Cauca y el $13 \%$ vive en otras ciudades. Ahora, la afiliación religiosa que predominó entre los y las participantes fue el catolicismo (57.3\%). Sin embargo, el $14,1 \%$ no profesa ninguna religión. En cuanto a los programas o carreras profesionales de las y los participantes, el 44,9\% estudia o estudió Psicología, seguido por Medicina (29,2\%). Además, el 71,4\% se encontraba aun estudiando uno de los programas mostrados en la Tabla 1 o es un(a) profesional que no cuenta con un posgrado.

En cuanto a la formación en temas de diversidad sexual y de género, el 53,5\% de los y las participantes se han capacitado en dichos temas. Predominantemente lo hicieron en instituciones educativas (26,5\%) y plataformas digitales (21,1\%). Asimismo, hubo participantes que se formaron en más de un lugar. No obstante, el $48,6 \%$ de los y las participantes no se formaron en temas de diversidad sexual y de género.

En cuanto a la experiencia clínica, el 67,6\% de la muestra tuvo menos de 4 años de experiencia. Esta experiencia se ha dado de forma predominante en hospitales (40\%) y clínicas (39,5\%). Asimismo, hubo participantes que han prestado atención clínica en más de un lugar. Finalmente, de manera relevante se destaca que más de la mitad de la muestra (57.8\%) atendió a, al menos, una persona trans o de género no binario

\section{Correlación entre Variables}

En la Tabla 2 se muestran las correlaciones entre las variables Atención Clínica, Disposición y Actitudes. Por otro lado, en la Tabla 3 se muestra la correlación entre las mismas variables mencionadas y Edad, la cual se segmentó en mayores de 30 y menores de 30.

Como se aprecia en la Tabla 2, la proporción de participantes que han atendido a pacientes trans y no presentan ningún prejuicio hacia esta población $(21,5 \%)$ es ligeramente menor a la de aquellos que no han atendido a personas de esta población (23,1\%). La proporción de aquellos/as que atendieron pacientes trans y presentan un prejuicio alto $(17,8 \%)$ es muy similar a la de aquellos/as participantes que no han atendido a pacientes trans y presentan también prejuicio alto hacia esta población $(17,9 \%)$. Las diferencias significativas no existen en esta instancia $(\mathrm{p}=0,96)$.

Tabla 2

Atención Clínica, Disposición y Actitudes hacia la comunidad trans.

\begin{tabular}{lcc}
\hline \multicolumn{1}{c}{ Variable } & $\begin{array}{c}\text { Sí ha atendido } \\
107(57,8 \%) \\
(\mathrm{n}=185)\end{array}$ & $\begin{array}{c}\text { No ha atendido } \\
78(42,2 \%) \\
(\mathrm{n}=185)\end{array}$ \\
\hline $\begin{array}{l}\text { Actitudes } \\
\text { Ningún prejuicio }\end{array}$ & $23(21,5)$ & $18(23,1)$ \\
Prejuicio bajo & $65(60,7)$ & $46(59,0)$ \\
Prejuicio alto & $19(17,8)$ & $14(17,9)$ \\
P valor & 0,96 & \\
Disposición & & \\
Ninguna & $75(70,1)$ & $50(64,1)$ \\
Moderada & $31(29,0)$ & $28(35,9)$ \\
Alta & $1(0,9)$ & $0(0,0)$ \\
P valor & 0,43 & \\
\hline
\end{tabular}

Respecto a la categoría Disposición, se aprecia que la proporción de los y las participantes que atendieron a personas trans y no presentan ninguna disposición hacia la población $(70,1 \%)$ es mayor a la de aquellos/as participantes que no han atendido a personas trans y no presentan ninguna disposición hacia esta población $(64,1 \%)$. Además, la proporción de participantes que no atendieron a personas trans y presentan una disposición moderada hacia esta población $(35,9 \%)$ es mayor en comparación a la de los y las participantes que han atendido a personas de esta población y presentan, igualmente, una disposición moderada hacia ellas (29\%). Finalmente, la proporción de participantes que han tenido pacientes trans y presentan una disposición $(0,9 \%)$ es ligeramente mayor a la proporción de quienes no han tenido pacientes trans y presentan también una disposición alta. Pese a esto, las diferencias no son significativas $(p=0,43)$.

Los resultados reflejan que, con respecto a la variable de Actitudes, la proporción de personas menores de 30 años que tuvieron prejuicios bajos hacia la población trans $(63,2 \%)$ es mayor que la proporción de aquellos/as con más de 30 años que presentaron prejuicios bajos hacia la población trans. Esta diferencia fue significativa $(p=0,04)$. 
Tabla 3

Edad, Actitudes y Disposición frente a la comunidad trans.

\begin{tabular}{lcc}
\hline \multicolumn{1}{c}{ Variable } & $\begin{array}{c}\text { Menores de } 30 \\
136(73,5 \%) \\
(\mathrm{n}=185)\end{array}$ & $\begin{array}{c}\text { Mayores de } 30 \\
49(26,5 \%) \\
(\mathrm{n}=185)\end{array}$ \\
\hline Actitudes & & \\
Ningún prejuicio & $24(17,6)$ & $17(34,7)$ \\
Prejuicio bajo & $86(63,2)$ & $25(51,0)$ \\
Prejuicio alto & $26(19,1)$ & $7(14,3)$ \\
P valor & 0,04 & \\
Disposición & & \\
Ninguna & $87(64,0)$ & $38(77,6)$ \\
Moderada & $49(36,0)$ & $10(20,4)$ \\
Alta & $0(0,0)$ & $1(2,0)$ \\
P valor & 0,03 & \\
\hline
\end{tabular}

En cuanto a la Disposición, el porcentaje de personas mayores de 30 años que no tuvieron una disposición hacia la comunidad trans $(77,6 \%)$ es mayor que el porcentaje de personas menores de 30 años que no presentaron disposición alguna $(63,2 \%)$, lo cual evidencia una diferencia significativa $(p=0,03)$. Por otro lado, el porcentaje de personas que han atendido personas trans y que no tuvieron una disposición hacia la comunidad trans $(70,1 \%)$ es mayor que el porcentaje de personas que no han atendido personas trans y que no presentaron disposición alguna hacia la población trans $(64,1 \%)$. Sin embargo, esta diferencia no es significativa $(p=0,43)$.

\section{DISCUSIÓN}

Este estudio tuvo como objetivo identificar la relación entre las actitudes, la disposición y la edad en la atención clínica a personas trans por parte de estudiantes universitarios colombianos. De esta manera, respecto a las actitudes, se encontró que los prejuicios que presentan en general los y las participantes son bajos, al igual que la disposición negativa. Esto quiere decir que no habría mayor probabilidad de discriminación hacia la población trans en este contexto; por el contrario, se estaría más cercano a la empatía.

Los resultados anteriores coinciden parcialmente con la mayoría de las investigaciones revisadas (Aylagas-Crespillo et al., 2017; Campo et al., 2014; Chárriez y Seda, 2015; Domínguez et al., 2018; Fajardo, 2017; Gleason et al., 2016; Lasso, 2014; Mejía y Benavides, 2008; Padilla et al., 2016; Palma y Orcasita, 2019; Profamilia, 2019; Ritterbusch et al., 2018;
Roberts y Fantz, 2014; Snelgrove et al., 2012). Cabe aclarar que, por mínimo que sea el porcentaje obtenido, el que un/a/e profesional de la salud tenga una actitud negativa afecta a todas las personas que sean atendidos por este/a/e profesional.

Según la teoría del comportamiento planificado de Azjen (1985), el principal factor que predice el cambio de conducta es la intención de cambiar. No obstante, esa intención está determinada por tres factores: las actitudes frente a la conducta, es decir, la valoración (positiva o negativa) que se hace sobre el comportamiento; la norma social, es decir, la presión social para llevar a cabo o no el comportamiento; y el control de acción, es decir, la autopercepción sobre ser capaz o no de llevar a cabo el comportamiento.

En este sentido, el comportamiento de la persona profesional de la salud o ciencias sociales variará de acuerdo a la valoración que tenga acerca de atender clínicamente a una persona trans o de género no binario; la presión social ejercida sobre este comportamiento, por ejemplo, la ética profesional de atender equitativamente a sus pacientes $\mathrm{y}$, por último, si considera que puede atenderles, teniendo en cuenta, por ejemplo, la religión que profese (factores externos) o sus habilidades para ello (factores internos).

Ahora, estos aspectos han sido reforzados y promovidos no solo por el contexto disciplinar y académico de los, las y les estudiantes y profesionales, sino también a nivel social. De esta manera, socialmente se han construido ideas sobre cómo deben comportarse los, las y les profesionales de la salud y ciencias sociales, y se exige una especie de congruencia y correspondencia con su área de estudio (Narro-Robles et al., 2004).

En ocasiones, las actitudes negativas entre los/las/les profesionales y sus pacientes trans pueden verse permeadas por sus respectivas representaciones sociales acerca de la identidad de género no binaria o trans. Lo anterior teniendo en cuenta que las representaciones sociales son aquellos conocimientos construidos a partir de las experiencias y la información disponible y que se transmiten a través de la educación, tradición y comunicación (Rodríguez y García, 2007).

En este sentido, las representaciones sociales de los/las/les profesionales pueden traducirse en 
actitudes negativas o positivas (Perales-Quenza y Vizcaíno-Gutiérrez, 2007). Sin embargo, si estas resultan ser negativas, pueden provocar como consecuencia que las personas trans opten por no dirigirse a los servicios de salud y se autoformulen y, por ende, pongan en riesgo su vida (Aylagas-Crespillo et al., 2017; Campo et al., 2014; Chárriez y Seda, 2015; Domínguez et al., 2018; Estrada y García, 2010; Fajardo, 2017; Gleason et al., 2016; Hoyos-Hernández y Valderrama, 2020; Mejía y Benavides, 2008; Lasso, 2014; Padilla et al., 2016; Palma y Orcasita, 2019; Profamilia, 2019; Ritterbusch et al., 2018; Roberts y Fantz, 2014; Snelgrove et al., 2012).

Además, se ha comprobado que cuando se tiene un acercamiento hacia un evento o situación en el que se aumente un sentimiento de agrado y bienestar, hay mayor probabilidad de que influya en el comportamiento hacia la situación en particular (Esteban et al., 2020). En este caso, lo anterior se enlaza a cómo los y las profesionales del estudio que sí han atendido a personas trans y han conocido su historia, tienden más a la empatía, a diferencia de los profesionales que no han tenido un acercamiento con esta población.

De esta manera, se pudo observar que el $57,8 \%$ de los y las participantes han atendido a población trans, lo que, al haber tenido una experiencia previa, puede generar una tendencia a aminorar prejuicios y disposiciones negativas. Esto puesto que han adquirido más conocimientos sobre las necesidades, procedimientos y problemáticas de la población mencionada, lo cual coincide con el estudio realizado por Ramos et al. (2018). En dicho estudio, los/las estudiantes de Medicina, al conocer la situación de las personas trans, tuvieron más intenciones de ofrecer servicios de salud hacia esta población.

En cuanto a identificar relaciones entre la edad, las actitudes y la disposición de los y las participantes, es importante recordar que se segmentó la edad en mayores y menores de 30 años. Así, se encontró que las personas menores de 30 años presentan mayores porcentajes en prejuicios $(82.3 \%$ ) hacia la población trans que las personas mayores de 30 años, quienes obtuvieron $65,3 \%$ respectivamente. Una de las posibles causas de estos resultados puede ser que las personas mayores de 30 años han tenido mayor experiencia brindando atención clínica a lo largo de su trayectoria profesional, a diferencia de los menores, quienes apenas pueden estar iniciando su ejercicio profesional. Por esto, se demuestra y coincide con algunos estudios en cuanto a que unos de los factores importantes son la experiencia en la práctica clínica y los conocimientos que se tengan respecto a las necesidades, particularidades y problemáticas de la población trans (Campo et al., 2014; Domínguez et al., 2018; Lasso, 2014; Mejía y Benavides, 2008; Profamilia, 2019; Sacsa, 2019).

Otra posible causa de que las personas mayores de 30 años hayan presentado menores niveles de prejuicios es debido a que su conducta puede estar regulada, principalmente, por las normas establecidas a la hora de ejercer su profesión. Esto permite que conozcan sus derechos y obligaciones tanto como profesionales como con la sociedad y las consecuencias que pueden acarrear al vulnerarlos, como recibir sanciones o incluso la pérdida de su tarjeta profesional (Narro-Robles et al., 2004).

De acuerdo con lo anterior, estos resultados dejan una interrogante sobre la educación que se le brinda a los/las/les profesionales de la salud en las instituciones de educación superior en temas de diversidad sexual y de género. Son pocas las instituciones que cuentan con pénsumes en los que se aborden temas de diversidad sexual y de género, lo cual concuerda con estudios realizados con profesionales de la salud en los que se menciona que durante la formación profesional no hubo contenidos que los/las/les capaciten para la atención de la población trans (Albuquerque et al., 2016; Builes et al., 2017; Chislom et al., 2018; Godoy et al., 2013; Farji, 2018; Francia-Martínez et al., 2017; Parkhill et al., 2014; Sacsa, 2019; Snelgrove et al., 2012; Ramos et al., 2018; Vance et al., 2015; Villasana et al., 2013; Vizcaíno et al., 2019; Zaldúa et al., 2015; Zapata et al., 2019).

Por otra parte, teniendo en cuenta la hipótesis postulada, la cual menciona que, a menor edad y trayectoria clínica, mayor disposición y actitudes positivas en los procesos de atención en salud a personas trans, y los resultados obtenidos, se halló que las personas mayores de 30 años tienen más actitudes y disposiciones favorables hacia la población trans, a diferencia de los menores de 30 años; por esto, dicha hipótesis se rechaza. De acuerdo con esto, no puede asumirse que el momento, contexto histórico o época en el que están creciendo cronológica, personal y profesional- 
mente los y las participantes sean variables determinantes para definir la calidad de atención clínica que ofrecen a las personas trans.

Ahora, para concluir, se tiene que, primero, las actitudes y disposiciones de la gran mayoría de los y las participantes no presentan prejuicios altos o disposición hacia la población trans. Sin embargo, este comportamiento puede variar según la valoración que se tenga acerca de atender clínicamente a una persona trans o de género no binario y otros factores asociados, como la presión social y el deseo de cambiar de comportamiento.

Se recomienda a los/las/les profesionales y futuros profesionales continuar con la actualización de conocimiento en el ámbito clínico, teniendo en cuenta los cambios sociales y la diversidad que habita en los seres humanos. Resulta necesario instruirse y formarse en temas de diversidades sexuales y de géneros de manera contante, y fortalecer dimensiones mas profundas e integrales que permitan ejercer su rol como profesionales, ciudadanos, cuidadores y-o agentes educadores en sus diferentes escenarios de socialización y desempeño.

Se recomienda a las instituciones de educación superior contar con planes de estudio más completos respecto a los temas claves sobre diversidades sexuales y de géneros. Algunos temas a desarrollar pueden ser: procesos de tránsitos de género, procedimientos de transformación corporal o expresiones de género no hegemónicas, fortalecimiento de actitudes de escucha, comunicación y atención transfirmativa y humanizada, mecanismos de exigibilidad de derechos, deconstrucción de prácticas clínicas e investigativas medicalizadas y patologizantes de las comunidades trans.

Como limitaciones principales de este estudio están: la primera corresponde al uso de un muestreo no aleatorio, dos, el uso de instrumentos de autoinforme, lo cual puede introducir sesgos de interpretación y tres, la influencia que pudo generar la deseabilidad social a la hora de responder el cuestionario, promoviendo quizás respuestas que se acercaran a un deber ser profesional para una mayor aprobación social.

Financiamiento: Este artículo se vincula al proyecto TranSER, el cual es financiado por el Ministerio de Ciencia Tecnología e Innovación (Convocatoria para Proyectos de
Ciencia, Tecnología e Innovación en Salud, 807 de 2018, código 125180764234), la Pontificia Universidad Javeriana, Cali, y el Centro de Estudios de Infectología Pediátrica, en alianza con Transmujer.

Conflicto de Intereses: No existen conflictos de intereses de parte de los autores de la investigación.

Aprobación de la Junta Institucional para la Protección de Seres Humanos en la Investigación: Comité de Ética en Investigación Biomédica de la Corporación Científica Pediátrica, Cali-Colombia.

Consentimiento o Asentimiento Informado: Todo participante completó un consentimiento informado.

Proceso de Revisión: Este estudio ha sido revisado por pares externos en modalidad de doble ciego.

\section{REFERENCIAS}

Albuquerque, G., Lima, C., Silva, G., Alves, M., Moreira, J., Figuereido, F., Silva, L., Barbosa, V., Silva, E., Engracia, V., De Abreu, L. \& Adami, F. (2016). Access to health fservices by lesbian, gay, bisexual, and transgender persons: systematic literature review. BMC International Health and Human Rights, 16(2), 1-10.

American Psychological Association [APA]. (2011). Respuestas a sus preguntas sobre las personas trans, la identidad de género y la expresión de género. Comunicaciones Públicas y de Afiliados de la APA, 1-6.

Antoniucci, M. (2016). El acceso a la salud de las personas trans: el caso del CADS de la ciudad de Mar del Plata [Tesis de pregrado, Universidad Nacional de Mar del Plata]. Humadoc. http://humadoc.mdp.edu.ar:8080/handle/123456789/537

Aylagas-Crespillo, M., García-Barbero, O. \& Rodríguez-Martín, B. (2017). Barriers in the social and healthcare assistance for transgender persons: A systematic review of qualitative studies. Enfermería Clínica, 28(4), 247-259.

Azjen, I. (1985). From intentions to action: A theory of planned behavior. En J. Kuhl \& J. Beckmann (Ed.), Action-control: Form cognition to behavior (pp.11-39). Springer.

Berredo, L. (2011). Dificultades Administrativas enfrentadas por las personas Trans en la Región Metropolitana de Chile [Tesis de pregrado, Universidad Academia Humanismo Cristiano]. Archivo digital. http://bibliotecadigital.academia.cl/handle/123456789/2188

Builes, M., Anderson, M. y Aragón, B. (2017). Devenir otro: transformaciones del terapeuta que atiende a parejas lesbianas y gais. Revista Colombiana de Psiquiatría, 46(1), 12-21.

Campo, A., Oviedo., H. y Herazo, E. (2014). Estigma y discriminación a profesional de la salud transgénero. Revista de la Facultad de Medicina, 62(1), 41-45. https://revistas.unal.edu.co/index.php/revfacmed/article/view/43693

Chárriez, M. y Seda, J. (2015). Los servicios de apoyo a las personas transgénero: Una realidad imperceptible. Revista Griot, 1(8), 7384. https://revistas.upr.edu/index.php/griot/article/view/1500 
Actitudes y Disposición de Estudiantes de la Salud y Ciencias Sociales en la Atención Clínica a Personas Trans

Chisolm, M., Willging, C., Daul, A., McNamara, S., Sante, S., Shattuck, D. \& Crandall, C. (2018). Transgender and gendernonconforming patients in the emergency department: What physicians know, think, and do. Annals of Emergency Medicine, 71(2), 183-188. $\quad$ https://doi.org/10.1016/j.annemergmed.2017.09.042

Colombia Diversa. (2010). Provisión de servicios afirmativos de salud para personas $L G T B$.

Domínguez, C., Ramírez, S. y Arrivillaga, M. (2018). Acceso a servicios de salud en mujeres transgénero de la ciudad de Cali, Colombia. MedUNAB, 20(3), 296-30. https://revistas.unab.edu.co/index.php/medunab/article/view/2404

Esteban, C., González-Rivera, J. A., Francia-Martínez, M. y Lespier, Z. (2020). Desarrollo de instrumentos para medir actitudes hacia la comunidad trans: Análisis preliminares. Revista Evaluar, 20(1), 64-78. https://revistas.unc.edu.ar/index.php/revaluar

Estrada, J. y García, A. (2010). Reconfiguraciones de género y vulnerabilidad el VIH/sida en mujeres transgénero en Colombia. Revista Gerencia en Políticas de Salud, 9(18), 90-102.

Fajardo, I. (2017). Acceso a los servicios de salud desde la perspectiva de las personas transgénero: influencias en la salud [Tesis de posgrado, Universidad Francisco de Quito]. Archivo digital. http://repositorio.usfq.edu.ec/handle/23000/6617

Farji, A. (2018). Los/as profesionales de la salud frente a la Ley de Identidad de Género Argentina. Tensiones entre el saber experto y el cuidado integral. Revista de Saúde Colectiva, 28(3), 118.

Fondo de Población de las Naciones Unidas [UNFPA]. (2019). Lineamientos de atención en los servicios de salud que consideran el enfoque diferencial de género y no discriminación para las personas LGBTI.

Francia-Martínez, M., Esteban, C. y Lespier, Z. (2017). Actitudes, conocimiento y distancia social de psicoterapeutas con la comunidad transgénero y transexual. Revista Puertorriqueña de Psicología, 28(1), 98-113.

Godoy, D., González, S. y Rivero, J. (2013). Componentes de formación para la atención en salud de personas sexo-género diversas. Comunidad y Salud, 11(1), 8-15.

Gleason, H., Livingston, N., Peters, M., Oost, K., Reely, E. \& Cochran, B. (2016). Effects of state nondiscrimination laws on transgender and gender nonconforming individuals perceived community stigma and mental health. Journal of Gay \& Lesbian Mental Health, 20(4), 1-25. https://www.tandfonline.com/doi/abs/10.1080/19359705.2016.1207582

Gonzáles, D. [La Disidencia]. (2020, 5 de marzo). Soy Isabella Castiblanco: Modelo, bailarina y trans [Archivo de vídeo]. Youtube. https://www.youtube.com/watch?v=qZdUwqLt1Qo

Gonzáles, D. [La Disidencia]. (2020, 31 de marzo). Coronavirus: población trans en medio de la emergencia sanitaria [Vídeo]. YouTube. https://www.youtube.com/watch?v=qZdUwqLt1Qo

Gonzáles, D. y Novoa, T. [La Disidencia]. (2019, 3 de julio). Se puede ser hombre sin pene [Vídeo]. YouTube. https://www.youtube.com/watch?v=t8Ko45Lm8O0
Gutiérrez, V. (2015). Actitudes de los estudiantes hacia el estudio. Caleidoscopio, 33, 140-156. https://doi.org/10.33064/33crscsh562

Hernández, R., Fernández, C. y Baptista, M. (2014). Metodología de la investigación (6ta. ed.). McGraw-Hill.

Hoyos-Hernández, P.A. y Concha, V. (2020). Representaciones sociales de estudiantes de posgrado en Psicología respecto a identidades trans. Sometido a Interdisciplinaria, Revista de Psicología y Ciencias Afines.

Hoyos-Hernández, P.A. y Valderrama, L.J. (2020). Necesidades en la atención en salud desde la perspectiva de las mujeres trans. En Ciclos de vida y vulnerabilidad social en el Valle del Cauca, Cali. Sello Editorial Javeriano.

Lancheros, L., Marconi, L., Manrique, M. y Mendivelso, M. (2007). Conceptos básicos acerca de las pruebas de actitud. Avances en Medición, 5, 163-167.

La Perra Roja, Z. [Las Igualadas]. (2019, 28 de mayo). Por estas razones ser mujer trans y lideresa es muy difícil - Zunga [Vídeo]. YouTube. https://www.youtube.com/watch?v=zon-4zKrE0w

Lasso, R. A. (2014). Transexualidad y servicios de salud utilizados para transitar por sexos-géneros. Revista CES Psicología, 7(2), 108-125. https://revistas.ces.edu.co/index.php/psicologia/article/view/2881

Losada, C. [La Pulla]. (2017, 31 de agosto). Los trans están condenados a morir jóvenes [Vídeo]. YouTube. https://www.youtube.com/watch?v=rOSdwVmgEK8

Lilienfeld, S. O., Lynn, S. J., Namy, L. L. y Woolf, N. J. (2011). Psicología: Una introducción. Pearson Education.

Mejía, A. y Benavides, L. (2008). Barreras de acceso a servicios de salud de las personas lesbianas, gay, bisexuales y transgénero de Bogotá D.C. http://www.sdp.gov.co/sites/default/files/barreras_acceso_servicios_salud_personas_lgbt_2008.pdf

Mendoza, Á. [La Disidencia]. (2019, 18 de septiembre). Aborto en personas trans [Vídeo]. YouTube. https://www.youtube.com/watch?v=VnFDeOa_M54

Ministerio de Salud [MinSalud]. (2016). Política de Atención Integral en Salud. https://www.minsalud.gov.co/sites/rid/Lists/BibliotecaDigital/RIDE/DE/modelo-pais-2016.pdf

Ministerio de Salud y Protección Social. (2005). Política Nacional de Prestación de Servicios de Salud. https://www.minsalud.gov.co/Ministerio/Documents/Politica\%20Nacional\%20de\%20Prestaci\%C3\%B3n\%20de\%20Servicios\%20de\%20Salud.pdf

Ministerio de Salud y Protección Social. (2014). Política Nacional en Sexualidad, Derechos Sexuales y Derechos Reproductivos del Ministerio de Salud y Protección Social. https://www.minsalud.gov.co/sites/rid/Lists/BibliotecaDigital/RIDE/DE/LIBRO\%20POLITICA\%20SEXUAL\%20SEPT\%2010.pdf

Narro-Robles, J., Rodríguez-Dominguez, J., Viesca-Treviño, C. y Abreu-Hernández, L. F. (2004). Ética y salud: retos y reflexiones. Gaceta médica de México, 140(6), 661-666. http://www.scielo.org.mx/pdf/gmm/v140n6/v140n6a17.pdf

Padilla, M., Rodríguez-Madera, S., Varas-Díaz, N. \& Ramos- 
Pibernus, A. (2016). Trans-Migrations: Border-Crossing and the Politics of Body Modification Among Puerto Rican Transgender Women. International Journal of Sexual Health, 28(4). 261277. https://dx.doi.org/10.1080\%2F19317611.2016.1223256

Palma, D. M. y Orcasita, L. T. (2019). Discursos de profesionales de la salud sobre la salud sexual de mujeres lesbianas y bisexuales. Interface, 23(15), 1-16. https://doi.org/10.1590/Interface. 170329

Parkhill, A., Mathews, J., Fearing, S. \& Gainsburg, J. (2014). A Transgender Health Care Panel Discussion in a Required Diversity Course. American Journal of Pharmaceutical Education, 78(4), 1-7. https://www.ncbi.nlm.nih.gov/pmc/articles/PMC4028590/

Perales-Quenza, C. J. y Vizcaíno-Gutiérrez, M. (2007). Las relaciones entre actitudes y representaciones sociales: elementos para una integración conceptual. Revista Latinoamericana de Psicología, 39(2), 351-361. http://pepsic.bvsalud.org/pdf/rlp/v39n2/v39n2a10.pdf

Profamilia y Estado del Reino de los Países Bajos [Profamilia]. (2019). ¿Cómo adaptar los servicios de salud sexual y reproductiva a las necesidades y circunstancias de las personas con experiencia de vida trans?

Ramos, J., Flores, A., Ramos, A., Rivera, E., Varas, N. y Rodríguez, S. (2018). Reduciendo el Estigma y Aumentando el Conocimiento Sobre las Necesidades de Salud de las Mujeres Transgénero: Intervención Piloto para Estudiantes de Medicina en Puerto Rico. Ciencias de la Conducta, 1(33). 51-74. https://cienciasdelaconducta.org/index.php/cdc/article/download/4/7

Ritterbusch, A., Correa, C y Correa, A. (2018). Stigma-related access barriers and violence against trans women in the Colombian healthcare system. Global Public Health, 13(12), 1831-1845. https://doi.org/10.1080/17441692.2018.1455887

Roberts, T. \& Fantz, C. (2014). Barriers to quality health care for the transgender population. Clinical Biochemistry, 47, 1-5. https://doi.org/10.1016/j.clinbiochem.2014.02.009

Rodríguez-Madera, S., Varas-Díaz, N., Neilands, T., Padilla, M. y Ramos-Pibernus, A. (2017). Proyecto Transforma. San Juan, Puerto Rico.

Rodríguez, T. y García, M. (2007). Representaciones sociales: teoría e investigación. Punto Cero, 21(33), 61-84. http://www.scielo.org.mx/pdf/sine/n36/n36a10.pdf

Rueda, M. [La Disidencia]. (2019, 17 de julio). Reconocerme me hizo amar la idea de querer vivir [Vídeo]. YouTube. https://www.youtube.com/watch?v=OyXHKESwkZ8\&t=1s

Sacsa, D. (2019). Competencias profesionales en la atención a usuarios de la diversidad sexual, en servicios de salud sexual y reproductiva de los distritos de Chorrillos Barranco y Surco 2018 [Tesis de posgrado, Universidad de San Martín de Porres]. Archivo digital. http://reposito-

rio.usmp.edu.pe/handle/20.500.12727/5218?show=full

Snelgrove, J., Jasudavisius, A., Rowe, W., Head, E. \& Bauer, R. (2012). Completely out-at-sea with two-gender medicine: A qualitative analysis of physician-side barriers to providing healthcare for transgender patients. BMC Health Serv Res, 110(2012). https://doi.org/10.1186/1472-6963-12-110

Ubillos, S., Páez, D. y Mayordomo, S. (2004). Actitudes: Definición y medición. Componentes de la actitud. Modelo de la acción razonada y acción planificada. En I. Fernández, S. Ubillos, E.M. Zubieta y D. Páez (Eds.). En Psicología Social, Cultura y Educación (pp. 301-326). Pearson Educación.

Vance, S., Halpern, B. \& Rosenthal, S. (2015). Health Care Providers' Comfort With and Barriers to Care of Transgender Youth. Journal of Adolescent Health, 56(2), 251-253. https://doi.org/10.1016/j.jadohealth.2014.11.002

Vargas, L., Vázquez, L. y Mogollón, A. (2010). Acceso a la atención en salud en Colombia. Revista de Salud Pública, 12(5), 701-712. http://www.scielo.org.co/pdf/rsap/v12n5/v12n5a01.pdf

Villasana, P., Sánchez, L. y Rojas, G. (2013). Percepción de docentes de la escuela de Medicina sede Aragua de la universidad de Carabobo sobre la atención en salud a población sexo-género diversa. Comunidad y Salud, 11(1), 51-57. https://www.redalyc.org/pdf/3758/375839306008.pdf

Vizcaíno, M., Montero, Y., Almenares, K. y Montero, Y. (2019). Percepción de estudiantes sobre la sexualidad como temática curricular en la carrera de medicina. Revista Cubana de Medicina Militar, 48(3), 597-611. http://www.revmedmilitar.sld.cu/index.php/mil/article/view/357

Williams-Colón, G. M., Alicea-Cruz, A. J., Pérez-Pedrogo, C. y Rodríguez-Gómez, J. R. (2021). Transfobia y religiosidad en Puerto Rico: Estudio exploratorio en profesionales de la salud. Revista Caribeña de Psicología, 5(1). https://doi.org/10.37226/rcp.v5i1.5633

Xavier, J., Bobbin, M., Singer, B. \& Budd, E. (2005). A need assessment of transgendered people of color living in Washington, DC. International Journal of Transgenderism, 8(23), 31-47. https://doi.org/10.1300/J485v08n02_04

Zaldúa, G., Longo, R., Lenta, M., Sopransi, M. y Joskowicz, A. (2015). Diversidades sexuales y derecho a la salud. Dispositivos, prácticas y desafíos en la exigibilidad. Anuario de Investigaciones, 22, 1-20. https://www.aacademica.org/maria.malena.lenta/142.pdf

Zapata, A., Díaz, K., Barra, L., Maureira, L., Linares, J. y Zapata, F. (2019). Atención de salud de personas transgéneros para médicos no especialistas en Chile. Revista Médica de Chile, 147(1), 65-73. https://scielo.conicyt.cl/pdf/rmc/v147n1/0717-6163-rmc147-01-0065.pdf 